\title{
ANÁLISIS COMPARATIVO DE LA GEOMETRÍA DE LA SECCIÓN TRANSVERSAL DE TIBIAS DE RESTOS HUMANOS DE PATAGONIA AUSTRAL Y CENTRO-NORTE DE MENDOZA
}

\author{
Jorge A. Suby ${ }^{1 *}$ y Paula Novellino²
}

\begin{abstract}
${ }^{I}$ INCUAPA-CONICET. Laboratorio de Ecología Evolutiva Humana. Departamento de Arqueología. Universidad Nacional del Centro de la Provincia de Buenos Aires. Quequén. Argentina

${ }^{2}$ CONICET-Museo de Ciencias Naturales y Antropológicas "J. C. Moyano”. Mendoza. Argentina
\end{abstract}

PALABRAS CLAVE Patagonia austral; Centro-Norte de Mendoza; geometría transversal; tibias; paleodieta

\begin{abstract}
RESUMEN El análisis de la geometría transversal de los huesos largos es un indicador de las fuerzas mecánicas a las que están sometidas las estructuras óseas, que refleja la actividad física, la salud nutricional y la variabilidad genética de los individuos. Aunque algunos análisis fueron realizados previamente en restos de individuos de la Patagonia austral, las conclusiones obtenidas parecen contradictorias respecto de la posible relación entre la morfología ósea de los huesos largos y la actividad física. El objetivo de este trabajo es profundizar en forma comparativa el análisis de la geometría de la sección transversal de las tibias de individuos de la Patagonia austral y el Centro-Norte de Mendoza, explorando la posible asociación entre el tipo de práctica económica realizada y el desarrollo de las estructuras óseas
\end{abstract}

de los miembros inferiores. Los resultados muestran que no existen diferencias significativas entre la robusticidad de los miembros inferiores de poblaciones de la Patagonia y del Centro-Norte de Mendoza. Sin embargo, al analizar los resultados de acuerdo con la dieta, los individuos con dietas marítimas en la Patagonia austral presentaron tibias más robustas que aquellos con dietas terrestres. En los individuos del Centro-Norte de Mendoza, los restos de individuos con dietas asociadas al consumo de maíz mostraron menor robusticidad en relación con otros individuos de esa región. Finalmente, sólo un caso de la Patagonia austral mostró resultados que podrían indicar que fue afectado por un trastorno metabólico de la salud. Rev Arg Antrop Biol 19(2), 2017. doi:10.17139/raab.2017.0019.02.01

\section{KEY WORDS Southern Patagonia; Cuyo; transversal geometry; tibias; paleodiet}

ABSTRACT Analysis of transversal geometry of long bones is an indicator of the work of mechanical forces over bone structures, informing about physical activity patterns, nutritional health, and genetic variability among individuals. Although some analyses were previously performed in human skeletons from southern Patagonia, contradictory conclusions were reported in regards to the relationship between long-bone morphology and physical activity patterns. The aim of this work is to comparatively increase the knowledge about the transversal geometry patterns in human remains of individuals from southern Patagonia and Center-North of Mendoza, studying the possible relation-

El análisis de la geometría transversal de huesos largos ha sido frecuentemente reconocido como uno de los indicadores de las fuerzas mecánicas a las cuales están sometidas las estructuras óseas (Pearson y Lieberman, 2004; Jurmain et al., 2012; Ruff y Larsen, 2014). Este tipo de investigaciones se deriva de modelos biomecánicos, según los cuales la geometría de la sección transversal de los huesos largos responde al patrón de cargas mecánicas que actúan sobre ellos (Ruff, 2008). Por lo tanto, es considerada, al menos en parte, un reflejo de las actividades físicas desarrolladas, asumiendo la relación entre las estructuras óseas y su función (Pearson y Buikstra, 2006). ship between economic practices and the structure of low limbs. The results showed no difference in the robusticity of the tibias between individuals from southern Patagonia and Center-North of Mendoza. However, individuals with maritime diets from Southern Patagonia showed more robusticity than individuals with terrestrial diets. Moreover, individuals from Center-North of Mendoza with cornbased diets showed lower robustiticy than other individuals from the same region. Finally, only one case from southern Patagonia showed results that could be related to some metabolic disease. Rev Arg Antrop Biol 19(2), 2017. doi:10.17139/raab.2017.0019.02.01

Otros factores han sido asociados a la geometría de los huesos largos, incluyendo patrones genéticos, el tamaño corporal y las proporciones de los miembros, cuyas relaciones han sido amplia-

\footnotetext{
*Correspondencia a: Jorge A. Suby. Laboratorio de Ecología Evolutiva Humana, Universidad Nacional del Centro de la Provincia de Buenos Aires. Calle $508 \mathrm{~N}^{\circ} 881$. 7631 Quequén. Argentina. E-mail: jasuby@conicet.gov.ar; jasuby@gmail.com
}

Financiamiento: Proyectos PICT0385 (J. Suby); PIP11220090100132 (P. Novellino).

Recibido 4 Mayo 2016; aceptado 22 Agosto 2016

doi:10.17139/raab.2017.0019.02.01 
mente documentadas (e.g. Trinkaus, 1981; Ruff y Hayes, 1982; Holliday, 1999; Stock, 2006). Además, debido a que factores dietarios, hormonales y nutricionales tienen también influencia sobre la morfología de las estructuras óseas (Pearson y Lieberman, 2004; Ruff, 2008), los análisis de la geometría de la sección transversal de huesos largos son además informativos de la salud metabólica de los individuos (Pearson y Lieberman, 2004; Brickley e Ives, 2008; Ruff, 2008).

Basados en estas asociaciones, los estudios de la geometría diafisaria de los huesos largos han sido abordados en numerosas investigaciones durante las últimas tres décadas, con el objetivo de analizar su relación con los patrones de adaptación morfológica esqueletal, incluyendo la evolución del sistema locomotor, cambios en la estructura esqueletal durante el desarrollo y crecimiento y los efectos de las estrategias de subsistencia sobre la morfología ósea (Ruff y Hayes, 1982, 1983; Ruff et al., 1984; Brock y Ruff, 1988; Larsen, 1995; Trinkaus y Ruff, 1999a, b; Bridges et al., 2000; Ledger et al., 2000; Stock y Pfeiffer, 2001; Holt, 2003; Rhodes y Knüsel, 2005; Weiss, 2005; Sládek et al., 2006; Stock, 2006; Nikita et al. 2011; Suby et al., 2011; ver Ruff y Larsen, 2014 para una revisión histórica). El mayor desarrollo de estas investigaciones producido durante las últimas dos décadas responde probablemente a la disponibilidad de sistemas informáticos automatizados, los cuales permiten la obtención de información mediante técnicas no destructivas, como la tomografía computada (O`Neill y Ruff, 2004). Al mismo tiempo, se ha demostrado que los análisis radiográficos y de los contornos morfológicos externos resultan útiles como buenas aproximaciones de la geometría de la sección transversal de los huesos largos en aquellos casos en los cuales los análisis tomográficos no pueden ser llevados a cabo debido a su costo, disponibilidad de las muestras o dificultad en su traslado (Stock y Shaw, 2007; Macintosh et al., 2013; Ruff y Larsen, 2014) ampliando el número de restos disponibles para análisis.

Contrariamente a esta tendencia, sólo han sido realizados unos pocos análisis biomecánicos de huesos largos de esqueletos arqueológicos pertenecientes a poblaciones nativas del sur de Sudamérica, todas ellas de Patagonia Austral. Pearson y Millones (2005) realizaron una evaluación de la posible adaptación al clima y las diferencias en la actividad física a partir del análisis morfológico externo de huesos largos de los miembros inferiores y superiores de restos esqueletales clasificados como Selk'nam y Yámanas, aunque sin detalles de procedencia y cronología de los restos. Los resultados reportados en ese trabajo indicaron que no habría diferencias significativas en relación a los índices de actividad física entre las muestras analizadas de ambas etnias.

Por su parte, Stock (2006) realizó una evaluación de la influencia del clima y la movilidad en la morfología de los huesos largos de cazadores-recolectores, en la cual se incluyó en forma comparativa una muestra no especificada de restos humanos de 34 esqueletos atribuidos a la etnia Yámana provenientes de Tierra del Fuego, individuos de América del Norte, sur de África y del sudeste de Asia. Se consideró en este caso que los Yámanas habitaron una región con clima frío y húmedo, con una baja o moderada movilidad terrestre y un terreno con relieves relativamente altos. Los resultados mostraron que los cazadores-recolectores de climas más fríos tienden a tener diáfisis de los huesos largos más robustas que aquellos de climas menos fríos. En contraste con Pearson y Millones (2005), sugieren que la movilidad y la actividad física tienen una relación positiva con la robusticidad de las diáfisis de las muestras estudiadas (Stock, 2006).

Por último, Suby (2007) realizó el análisis de la geometría de la sección interna de la tibia sobre un pequeño número de esqueletos de $\mathrm{Pa}$ tagonia Austral, estudiados a través de imágenes tomográficas, que mostraron una tendencia a la mayor robusticidad en los individuos con dietas marítimas, en concordancia con los resultados propuestos por Stock (2006). Similares resultados obtuvieron Suby y Guichón (2009) en un análisis de la robusticidad femoral de esqueletos humanos de Patagonia Austral, basado en mediciones óseas externas.

El objetivo de este trabajo es explorar la posible asociación entre el tipo de práctica económica desarrollada por individuos cazadoresrecolectores de Patagonia Austral y la geometría de la sección transversal de las tibias como aproximación a la actividad física desarrollada. A su vez, se analizan los resultados en forma 
comparativa con los alcanzados sobre los mismos indicadores en una muestra de restos humanos provenientes del Centro-Norte de Mendoza (región de Cuyo), cuyas características dietarías se basan en un significativo aporte de maíz (Gil et al., 2009).

\section{Muestra y contexto arqueológico}

La muestra analizada está compuesta por individuos adultos de ambos sexos, considerando adultos aquellos con una edad estimada superior a los 18 años al momento de la muerte. Los individuos subadultos fueron excluidos, de manera de evitar inconsistencias basadas en el desarrollo óseo incompleto. Sólo fueron incluidos individuos que tuvieran los elementos a analizar completos y en buenas condiciones de conservación. Además sólo se tuvieron en cuenta restos que tuvieran información de isótopos estables, de manera de evaluar las posibles diferencias asociadas a la actividad económica sobre las estructuras óseas.
Se estudiaron en total 14 esqueletos, 7 de los cuales provienen de Patagonia Austral y 7 al Centro-Norte de Mendoza. De los restos de Patagonia Austral, 4 son esqueletos masculinos y 3 femeninos. A su vez, 4 son adultos jóvenes y los restantes 3 son adultos medios. De los esqueletos provenientes del Centro-Norte de Mendoza, 3 pertenecen a individuos masculinos y 4 a individuos femeninos, de los cuales 1 es adulto joven y los restantes adultos medios (Tabla 1). En todos los casos se tuvieron en cuenta los datos sexo-etarios y paleodietarios consignados por los autores que se mencionan en la Tabla 1.

Los cambios en las estrategias de subsistencia han sido asociadas a modificaciones en los patrones de movilidad, la actividad física y la salud, aspectos que han sido documentados en el caso de la adopción de la agricultura en diversos grupos pre-hispánicos de América (e.g. Kelly, 1992; Buikstra y Milner, 1991; Larsen, 1995; Bridges, 2000). En tal sentido, en este trabajo consideramos la información paleodietaria como un proxy de las estrategias de subsisten-

TABLA 1. Esqueletos humanos analizados, correspondientes a sitios arqueológicos de Patagonia Austral y Centro-Norte de Mendoza

\begin{tabular}{|c|c|c|c|c|c|c|c|c|}
\hline Región & Sitios & Fechado & Sexo & Edad & $\delta^{13} \mathrm{Ccol}$ & $\delta^{15} \mathrm{~N}$ & Dieta & Referencia \\
\hline \multirow{7}{*}{$\begin{array}{c}\text { Patagonia } \\
\text { Austral }\end{array}$} & Cabo Vírgenes 17 & $900 \pm 40$ & M & $20-34$ & $-16,83$ & 11,4 & Mix & L'Heureux et al., 2003 \\
\hline & Orejas de Burro 2 & $3565 \pm 45$ & M & $45-50$ & $-18,5$ & 13,7 & $\mathrm{~T}$ & $\begin{array}{l}\text { L'Heureux y Barberena, } \\
2008\end{array}$ \\
\hline & La Arcillosa 2 & $5208 \pm 58$ & $\mathrm{~F}$ & $20-24$ & 17,9 & 13,1 & $\mathrm{~T}$ & Santiago et al., 2011 \\
\hline & Las Mandíbulas & Moderno & M & $20-24$ & $-18,8$ & 11,5 & $\mathrm{~T}$ & Guichón et al., 2000 \\
\hline & Puesto Pescador 1 & $335 \pm 35$ & M & $21-25$ & $-20,3$ & 12,5 & $\mathrm{~T}$ & Suby et al., 2008 \\
\hline & Paiashauaia & $1504 \pm 46$ & $\mathrm{~F}$ & $35-45$ & $-11,9$ & - & $\mathrm{Ma}$ & Suby et al., 2011 \\
\hline & Caleta Falsa S7esq2 & $820 \pm 40$ & $\mathrm{~F}$ & $30-39$ & $-11,8$ & 18,5 & Ma & Guichón y Suby, 2011 \\
\hline \multirow{7}{*}{$\begin{array}{c}\text { Centro } \\
\text { Norte } \\
\text { de } \\
\text { Mendoza }\end{array}$} & Tumulo II - 239 & $1269 \pm 35$ & $\mathrm{~F}$ & 50 & $-11,9$ & 9,6 & RM & Gil et al., 2009 \\
\hline & Tumulo II -243 & $1178 \pm 41$ & $\mathrm{~F}$ & $35-49$ & $-12,1$ & 10,1 & $\mathrm{RM}$ & Gil et al., 2009 \\
\hline & Viluco San Carlos - 1197 & $208 \pm 38$ & M & $40-45$ & $-17,2$ & 10,3 & PM & Gil et al., 2009 \\
\hline & Las Barrancas Y2 -1263 & $2084 \pm 40$ & M & 45 & $-18,0$ & 9,9 & PM & Gil et al., 2009 \\
\hline & San Carlos - 1221 & $142 \pm 41$ & M & $40-49$ & $-18,7$ & 8,4 & PM & Gil et al., 2009 \\
\hline & San Carlos - 1223 & $177 \pm 34$ & $\mathrm{~F}$ & $19-23$ & $-17,9$ & 10,7 & PM & Gil et al., 2009 \\
\hline & Capiz Alto - CA2 & Contacto & $\mathrm{F}$ & $40-45$ & $-14,9$ & 11,7 & PM & Gil et al., 2009 \\
\hline
\end{tabular}

Referencias: M:masculino; F:femenino; Mix:mixta; T:terrestre; Ma:marítima; RM:rica en maíz, correspondientes a la región montañosa occidental; PM:pobre en maíz, correspondientes a la planicie oriental. 
cia, y por lo tanto con las posibles demandas mecánicas sobre los miembros inferiores que podrían estar asociadas a ellas.

Los restos provenientes de Patagonia Austral corresponden a esqueletos recuperados en la región de Magallanes y costa atlántica de Santa Cruz, al norte de Tierra del Fuego, a la región del Canal Beagle y al extremo sur oriental de Tierra del Fuego (Fig. 1). Evidencias etnográficas proveen información de dos principales grupos de cazadores-recolectores: terrestres en el conti- nente y norte de Tierra del Fuego, con dietas basadas fundamentalmente en el consumo de guanaco (Lama guanicoe), y cazadores-recolectores marítimos en el sur de Tierra del Fuego, especializados en la explotación de recursos marinos a través del uso de canoas para la navegación del archipiélago costero (e.g. Hyades y Deniker, 1891; Gusinde, 1982, 1986; Orquera y Piana, 1999, 2009). Sin embargo, los datos arqueológicos y paleodietarios sugieren escenarios más complejos, no completamente coincidentes con
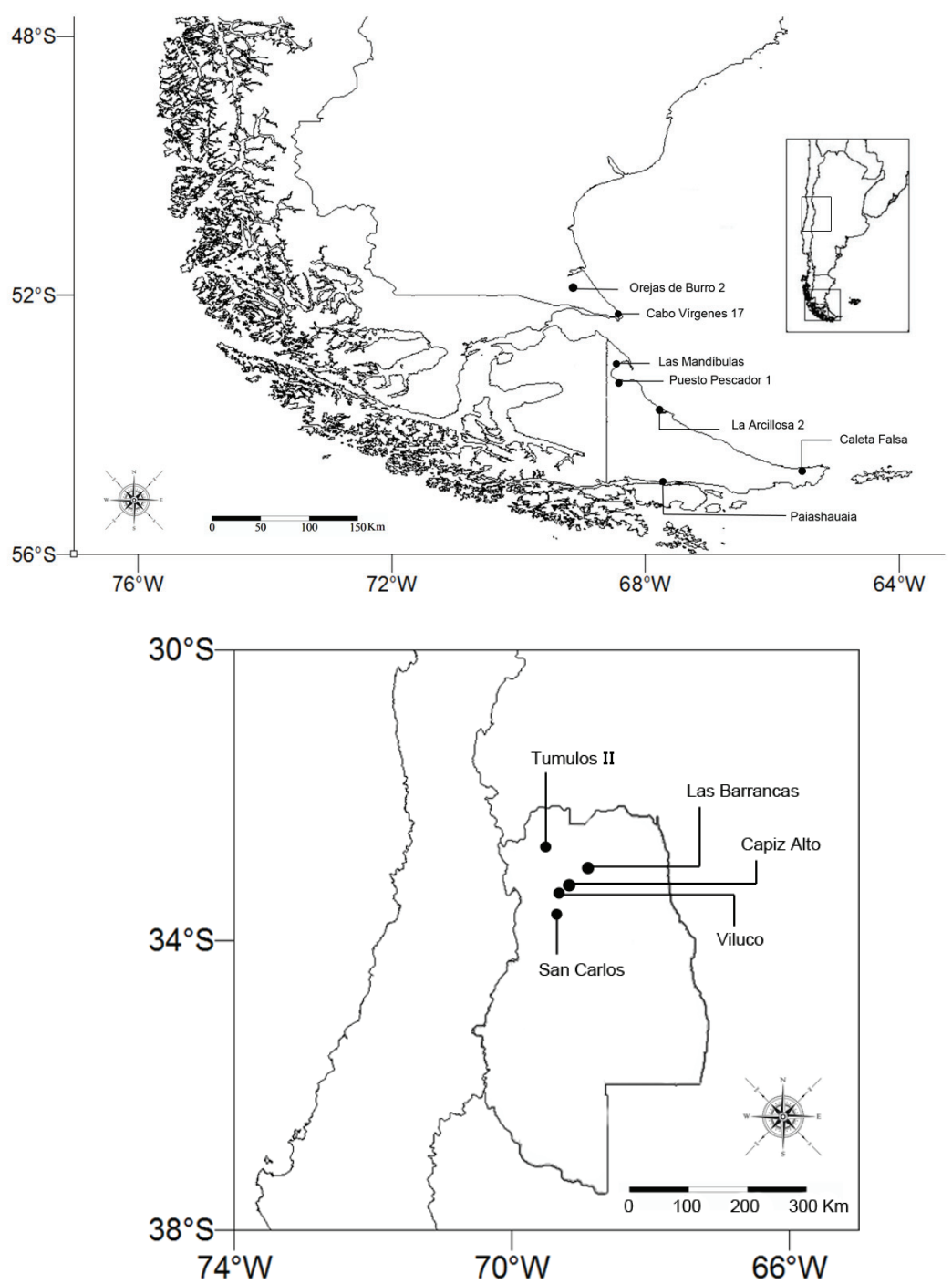

Fig. 1. Localización geográfica de los restos analizados procedentes de Patagonia Austral (arriba) y Centro-Norte de Mendoza (abajo) de los sitios arqueológicos analizados en el curso inferior del río Colorado. 
los registros etnográficos e históricos. Los estudios isotópicos realizados muestran que en el caso del extremo continental el consumo de proteínas de origen marino está presente hasta una distancia de $90 \mathrm{~km}$ de la costa, mientras que en el estrecho de Magallanes esta distancia se reduce a $20 \mathrm{~km}$ (Borrero y Barberena, 2006; Morello et al., 2012). De manera similar, en el norte de Tierra del Fuego se evidencia el consumo al menos estacional de recursos marítimos en sitios próximos a la costa, mientras el patrón dietario se torna más terrestre en el interior (Barberena, 2002). Por el contrario, el consumo de recursos marinos es más acentuado en muestras del sur de Tierra del Fuego, en relación a las analizadas en la región continental y el norte de Tierra del Fuego (e.g. Barberena, 2002; Yesner et al., 2003, Panarello et al., 2006; Zangrando, 2009; Santiago et al., 2011).

Desde un punto de vista biológico, estudios basados en ADN mitocondrial sugieren que los grupos de la región oriental de Santa Cruz y los grupos de Tierra del Fuego comparten ancestros comunes (Lalueza et al., 1997). Sin embargo, cierto grado de variabilidad cráneofacial fue observada, tendiendo a agrupar los grupos fueguinos por un lado y los continentales por otro (Cocilovo y Guichón, 1986; González-José et al., 2002) y mayor proximidad entre Yámanas y Kaweshkar que los Selk'nam y estos últimos (Varela et al., 1993-94; Guichón, 1994). Las causas de esta diferenciación permanecen poco claras, aunque algunos análisis sugieren que el desarrollo de estrategias dietarias y el aislamiento de Tierra del Fuego como resultado de la apertura del Estrecho de Magallanes podrían haber tenido un rol significativo en este proceso evolutivo (González-José et al., 2002; Perez et al., 2007).

Los restos provenientes de la región del Centro-Norte de Mendoza, por su parte, corresponden a individuos recuperados entre $30^{\circ} \mathrm{S}$ y $32^{\circ} \mathrm{S}$, en la provincia de Mendoza, Argentina (Fig. 1). Esta región, de clima templado, árido a semiárido y con precipitaciones anuales que promedian los $250 \mathrm{~mm}$ (Gil et al., 2009), está caracterizada por dos áreas con relieves claramente diferentes: una montañosa al oeste y otra con relieves relativamente planos formando una planicie al este (González Díaz y Fauqué, 1993; Gil et al., 2009). Los esqueletos incluidos en este trabajo incluyen 2 individuos que fueron recuperados en la región montañosa (Túmulo II-239 y Túmulo II-243) y los restantes pertenecen a la planicie oriental (Menéndez et al., 2014). Un análisis detallado de las características arqueológicas asociadas a estos hallazgos fue presentado por Gil et al. (2009).

Se ha propuesto a partir de evidencia arqueológica e histórica que las poblaciones humanas que ocuparon la región de Cuyo durante el Holoceno tardío se caracterizaron por una economía agricultora que adoptó el cultivo de maíz (Novellino et al., 2004; Gil et al., 2009). La antigüedad de la explotación del maíz por estas poblaciones es motivo de controversia, aunque al menos ha estado presente durante los últimos 2000 años, periodo al cual corresponden los esqueletos incluidos en este trabajo. Los resultados paleodietarios a partir de isótopos estables sobre estos esqueletos indican que el maíz fue consumido en forma significativa con posterioridad a los 1250 años AP, con una disminución durante los últimos 300 años AP (Gil et al., 2009). Respecto de la variabilidad dietaria en términos espaciales, los restos provenientes de la región montañosa occidental muestran mayores valores de $\delta^{13} \mathrm{Ccol}$ (Tabla 1 ), lo que podría asociarse a una dieta enriquecida en maíz en relación a los esqueletos provenientes de la región de planicie oriental (Gil et al., 2009). Estos resultados modifican los presentados previamente por Gil (2003) y Gil et al. (2006).

\section{Metodología}

Se analizó la geometría de la sección transversal de tibias de individuos de Patagonia Austral y el Centro-Norte de Mendoza. Se seleccionó este elemento dado que está más afectado por los patrones asociados a la actividad física, a diferencia del fémur en el cual la sección transversal está más relacionada con la forma corporal y la estructura de la pelvis (Stock, 2006; Ruff, 2008).

Para este trabajo cada tibia fue estudiada mediante tomografía computada (TC) empleando un tomógrafo computado Philips brilliance 64-slices en el Instituto Radiológico Mar del Plata (Provincia de Buenos Aires). El protocolo técnico incluyó imágenes de $1 \mathrm{~mm}$ 
de espesor adquiridas a $100 \mathrm{kv}$ y $80 \mathrm{~mA}$, con una matriz de resolución de $512 \times 512$ y campo de imagen de $418 \mathrm{~mm}$. Las tibias fueron orientadas y fijadas en posición anteroposterior, con su eje longitudinal coincidiendo con el del tomógrafo, siguiendo los procedimientos propuestos por Ruff y Hayes (1983) y reproducidos en Ruff (2008). Las imágenes tomográficas fueron obtenidas al $35 \%$ y $65 \%$ de la longitud biomecánica (i.e. la media entre las distancia desde centro de la superficie articular tibio-astragalina al cóndilo medial y al cóndilo lateral proximal de la tibia, sensu White et al., 2012) de las tibias, tomando la epífisis proximal como $100 \%$, regiones donde la geometría está menos influida por las dimensiones de la epífisis (Trinkaus y Ruff, 1999a).

A partir de las imágenes de TC, se calcularon indicadores de la rigidez mecánica asociados a las diferentes fuerzas que afectan a las tibias, mediante el software Image $J$ versión 1.49 (http://rsbweb.nih.gov/ij/download. html). Se calculó el área cortical (CA) como indicador de la rigidez y resistencia a las fuerzas de compresión y tensión puras cuando los huesos son cargados en su eje longitudinal. Además, se calculó el área total subperióstica (TA), la cual incluye el área por debajo del perímetro externo de la sección transversal y refleja la distribución relativa de hueso en esa sección (Ruff, 2008). Sin embargo, los huesos largos normalmente reciben cargas por fuera de su eje central, por lo que las fuerzas de compresión y tensión puras son inusuales. Por lo tanto, CA y TA son considerados indicadores de interés morfológico más que biomecánico y por esta razón resultan más apropiados para inferir la salud metabólica de los individuos que su actividad física (Ruff et al., 1993; Ruff, 2008). Por el contrario, las fuerzas de flexión y torsión son más frecuentes en los huesos largos. Para analizar la influencia de este tipo de fuerzas sobre las estructuras óseas se calcularon el segundo momento de área (I) en sus ejes mínimo (Imin) y máximo (Imax), los cuales estiman la resistencia a las fuerzas de flexión (Ruff, 2008); y el momento de inercia $(\mathrm{J})$, calculado como la suma aritmética del Imin y el Imax. El momento de inercia (J) constituye un indicador de la rigidez torsional en dos planos perpendiculares, en este caso los planos de mayor y menor carga mecánica. Incrementos de I y J con áreas óseas similares sugieren patrones de mayor actividad física, las cuales en condiciones fisiológicas normales podrían resultar en secciones transversales de mayor rigidez (Ruff, 2008).

Dado que la resistencia de los huesos largos está influida por su longitud, las comparaciones de propiedades biomecánicas estructurales entre individuos deben ser estandarizadas. Debido a que los elementos distales de los miembros superiores e inferiores (i.e. radio-cúbito y tibia-peroné, respectivamente) se encuentran menos afectados por el tamaño corporal que los elementos proximales (i.e. húmero $\mathrm{y}$ fémur) (Ruff, 2008), pueden ser estandarizados por la longitud ósea. Aunque algunas controversias se han planteado acerca de la forma más apropiada de estandarizar los indicadores biomecánicos, en este trabajo, al igual que otros realizados previamente (e.g. Bridges et al., 2000; Ledger et al., 2000), se siguió la propuesta de Ruff et al. (1993), quienes sugieren que CA y TA pueden ser estandarizadas al dividirlas por la fórmula longitud $o_{s e a^{3}} \times 10^{8}$, mientras I y J deben dividirse por la fórmula longitud ósea $a^{5.33} \times 10^{12}$. Para estas estandarizaciones se midió la longitud biomecánica de las tibias mediante una tabla osteométrica (Ruff y Hayes, 1983; Trinkaus y Ruff, 1999a, b). Todos los indicadores fueron calculados para ambas tibias cuando se encontraron disponibles, tomándose el promedio aritmético como resultado final.

Todos los resultados fueron estudiados para el conjunto total de la muestra de cada región y posteriormente clasificados de acuerdo a su dieta. No se exploró la influencia de la variabilidad sexual en la robusticidad de las tibias debido al reducido tamaño muestral. En el caso de Patagonia se clasificaron en dietas con predominio de recursos terrestres, marítimos y mixtas, considerando como mixtos aquellos en los cuales no existe un predominio claro de recursos marítimos o terrestres en la dieta (Borrero y Barberena, 2006). Los restos de Mendoza se clasificaron en aquellos con mayor y menor consumo de maíz en base a los análisis paleodietarios, los primeros provenientes de la región montañosa occidental 
y los segundos de la planicie oriental, los primeros con mayor consumo de maíz en la dieta que la segunda (Gil et al., 2009). El Análisis de la varianza (ANOVA) se realizó en todos los casos, mientras que las correlaciones de Pearson fueron calculadas entre CA y TA para evaluar la asociación entre estas. El valor de significancia fue establecido en $p \leq 0,05$ en todos los análisis.

\section{RESULTADOS}

Los resultados de los valores biomecánicos calculados son presentados en su totalidad en las Tablas 2 y 3 , para las mediciones realizadas sobre el $35 \%$ y $65 \%$ de las tibias, respectivamente. En ninguno de los casos los valores medios al- canzados en los restos de Patagonia y el CentroNorte de Mendoza resultaron significativamente diferentes. La asociación entre CA y TA fueron significativas en los restos de ambas secciones óseas (Fig. 2), con tendencias similares aunque sin diferencias entre regiones. Sin embargo, se observó una mayor propensión de mayores valores de CA en relación a TA en los restos de Patagonia respecto de los de Mendoza en ambas secciones de la tibia (Fig. 2). En el caso de Patagonia, los dos individuos con dietas marítimas analizados presentaron mayores valores de $\mathrm{CA}$ y TA que aquellos individuos con dietas terrestres y mixtas. Sin embargo, en uno de los individuos se observó un menor valor de CA respecto de TA; es decir, este presenta una menor superficie de hueso cortical en relación a individuos con

TABLA 2. Valores biomecánicos estandarizados de la sección transversal de tibias al 35\% de su longitud

\begin{tabular}{cccccc}
\hline Sitios & TA & CA & Imax & Imin & J \\
\hline Cabo Vírgenes 17 & 765,33 & 718,44 & 338,95 & 166,23 & 505,18 \\
Orejas de Burro 2 & 834,26 & 759,62 & 471,97 & 216,77 & 688,74 \\
La Arcillosa 2 & 1002,41 & 821,66 & 685,67 & 322,71 & 1008,38 \\
Las Mandíbulas & 904,29 & 737,46 & 520,19 & 229,40 & 749,60 \\
Puesto Pescador 1 & 886,84 & 794,72 & 422,34 & 236,43 & 658,77 \\
Paiashauaia & 1417,81 & 783,75 & 981,49 & 589,11 & 1570,60 \\
Caleta Falsa S7.esq2 & 1190,95 & 942,56 & 720,21 & 385,08 & 1105,29 \\
\hline \multicolumn{1}{c}{ Media } & 1044,11 & 810,59 & 567,06 & 284,94 & 852,00 \\
Tumulo II - 239 & 245,87 & 83,14 & 214,77 & 146,87 & 359,33 \\
\hline Tumulo II -243 & 720,18 & 481,08 & 282,74 & 184,22 & 466,97 \\
Viluco San Carlos - 1197 & 811,77 & 587,79 & 373,04 & 220,95 & 594,00 \\
Las Barrancas Y2 -1263 & 983,87 & 712,03 & 388,82 & 407,56 & 796,38 \\
San Carlos - 1221 & 1077,65 & 753,77 & 588,67 & 409,94 & 998,61 \\
San Carlos - 1223 & 1250,76 & 853,46 & 671,89 & 645,78 & 1317,67 \\
Capiz Alto - CA2 & 1017,26 & 717,60 & 424,10 & 392,33 & 816,44 \\
\hline \multirow{2}{*}{ Media } & 1101,28 & 789,10 & 403,83 & 643,20 & 1047,04 \\
\hline & 1086,17 & 765,19 & 495,46 & 499,77 & 995,23 \\
\hline
\end{tabular}

Referencias: TA:área total subperióstica; CA:área cortical; Imax e Imin: segundo momento de área en sus ejes mínimo y máximo; J:momento de inercia. 
TABLA 3. Valores biomecánicos estandarizados de la sección transversal de tibias al $65 \%$ de su longitud

\begin{tabular}{crrrrr}
\hline Sitios & TA & CA & Imax & Imin & J \\
\hline Cabo Vírgenes 17 & 1422,30 & 1070,89 & 1556,71 & 575,18 & 2131,88 \\
Orejas de Burro 2 & 1391,08 & 1093,51 & 774,66 & 225,44 & 1000,09 \\
La Arcillosa 2 & 1590,13 & 1291,78 & 1877,13 & 783,77 & 2660,91 \\
Las Mandíbulas & 1615,78 & 1148,79 & 1805,70 & 564,10 & 2369,79 \\
Puesto Pescador 1 & 1494,18 & 1245,56 & 1482,69 & 486,85 & 1969,54 \\
Paiashauaia & 1576,58 & 803,31 & 994,03 & 466,86 & 1460,89 \\
Caleta Falsa S7.esq2 & 1810,56 & 1358,12 & 1763,57 & 651,61 & 2415,17 \\
\hline Media & 1593,16 & 1177,92 & 1371,20 & 496,11 & 1867,31 \\
ds & 165,25 & 194,31 & 474,21 & 196,75 & 660,98 \\
\hline Tumulo II - 239 & 991,42 & 674,76 & 700,81 & 268,64 & 969,45 \\
Tumulo II -243 & 1111,04 & 750,18 & 786,47 & 368,45 & 1154,92 \\
Viluco San Carlos - 1197 & 1529,75 & 1014,93 & 999,56 & 926,87 & 1926,43 \\
Las Barrancas Y2 -1263 & 1383,34 & 939,67 & 964,85 & 328,47 & 1293,32 \\
San Carlos - 1221 & 1522,41 & 1130,25 & 1849,45 & 954,08 & 2803,53 \\
San Carlos - 1223 & 1776,09 & 1229,18 & 1221,87 & 685,52 & 1907,39 \\
Capiz Alto - CA2 & 1549,52 & 1030,04 & 939,48 & 1083,86 & 2023,34 \\
\hline Media & 1552,22 & 1068,81 & 1195,04 & 795,76 & 1990,80 \\
\hline & 141,45 & 112,45 & 382,58 & 298,23 & 538,32 \\
\hline
\end{tabular}

Referencias: TA:área total subperióstica; CA:área cortical; Imax e Imin: segundo momento de área en sus ejes mínimo y máximo; J:momento de inercia.

superficie total similar (Fig. 2). Esta reducción del espesor cortical puede ser atribuido a una patología sistémica, probablemente metabólica, previamente sugerida en este individuo, y que implicó modificaciones en la morfología normal de las tibias (Suby et al., 2011).

$\mathrm{Al}$ analizar las variables biomecánicas Imax, Imin y J no se observaron diferencias significativas entre los individuos de Patagonia y Mendoza en ambas secciones de las tibias. (Figs. 3 y 4 ). Sin embargo, cuando se estudiaron los valores de cada grupo de individuos de acuerdo a su dieta, se observó una tendencia a mayores valores en los individuos con dietas predominantemente marítimas que aquellos con dietas terrestres y mixtas en la región del 35\% de las tibias (Fig. 3 ), aunque no significativamente diferentes. En el caso de los restos de Mendoza, aquellos pro- cedentes de la región oeste, con un mayor consumo de maíz, presentaron valores biomecánicos significativamente menores en ambas porciones óseas analizadas respecto de los individuos de la planicie oriental de Mendoza, asociada a un menor consumo de maíz (Figs. 3 y 4).

\section{DISCUSIÓN Y CONCLUSIONES}

Los estudios de la geometría transversal de huesos largos permiten construir algunas hipótesis acerca de posibles variaciones en la actividad física asociadas al tipo de economía desarrollada en cazadores-recolectores del sur de Sudamérica. Aunque los tamaños muestrales son reducidos, se incluyen aquí individuos que no fueron analizados previamente por Suby y Guichón (2009) a partir de mediciones femorales externas, lo que 

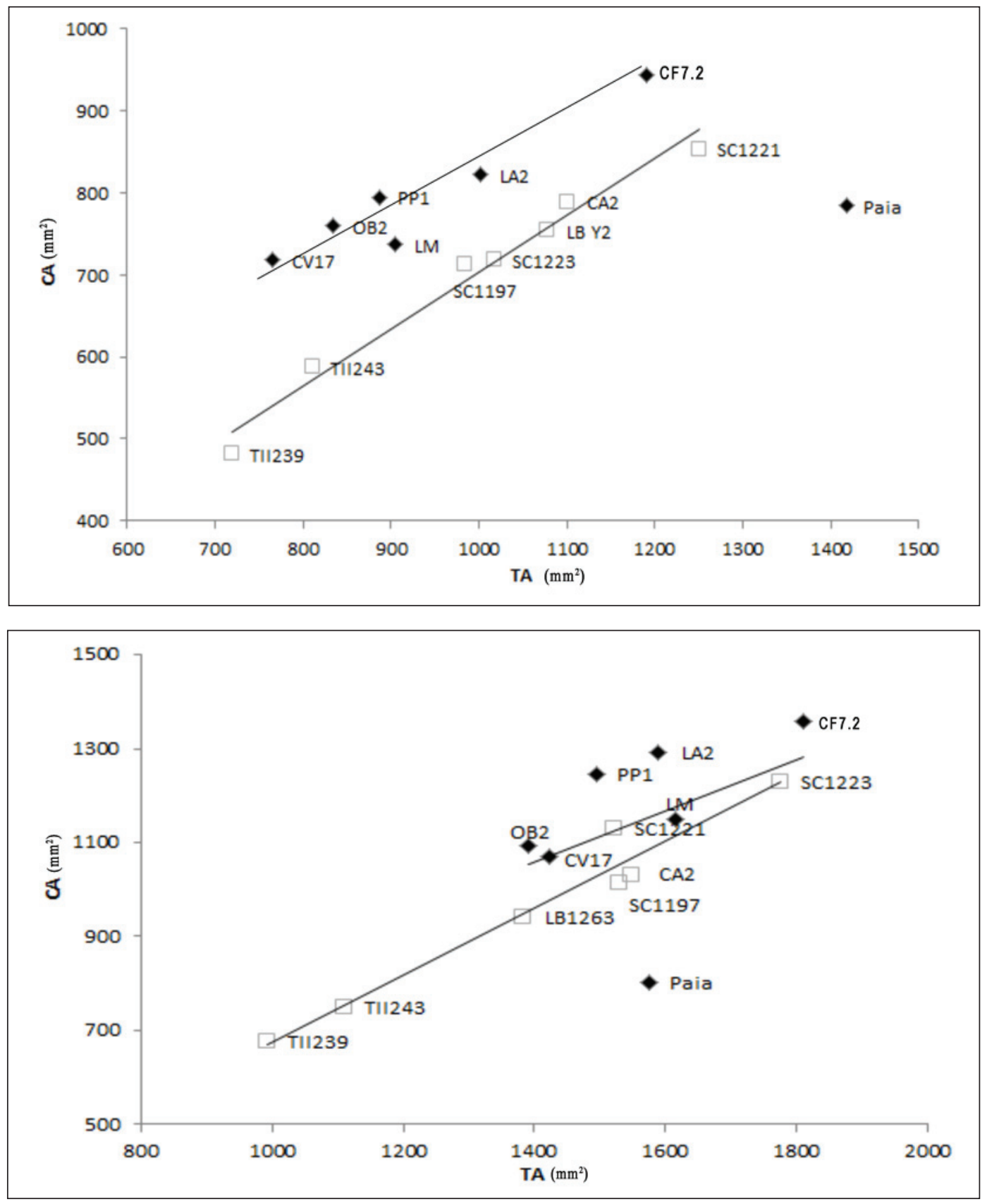

Fig. 2. CA vs TA (en $\mathrm{mm}^{2}$ ) en el 35\% (arriba) y 65\% (abajo) de las tibias analizadas. Patagonia: 35\% Pearson $(\mathrm{r}=0,83 \mathrm{p}<0,05) ; 65 \%$ Pearson $(\mathrm{r}=0,79 \mathrm{p}<0,05)$; Mendoza: 35\% Pearson $(\mathrm{r}=0,84 \mathrm{p}<0,05)$; 65\% Pearson $(\mathrm{r}=0,8$ $\mathrm{p}<0,05)$.

podría interpretarse como evidencias independientes a las reportadas anteriormente. En el caso de los restos del Centro-Norte de Mendoza, sólo son analizados dos individuos con mayor consumo de maíz de la región montañosa, por lo que, al igual que para los restos de Patagonia Austral, y a pesar de que los análisis estadísticos realizados reportaron resultados significativos en algunos casos, los resultados deben ser interpretados como tendencias que podrán ser puestas a prueba en el futuro a través de análisis más exhaustivos y sobre conjuntos de mayor tamaño. 


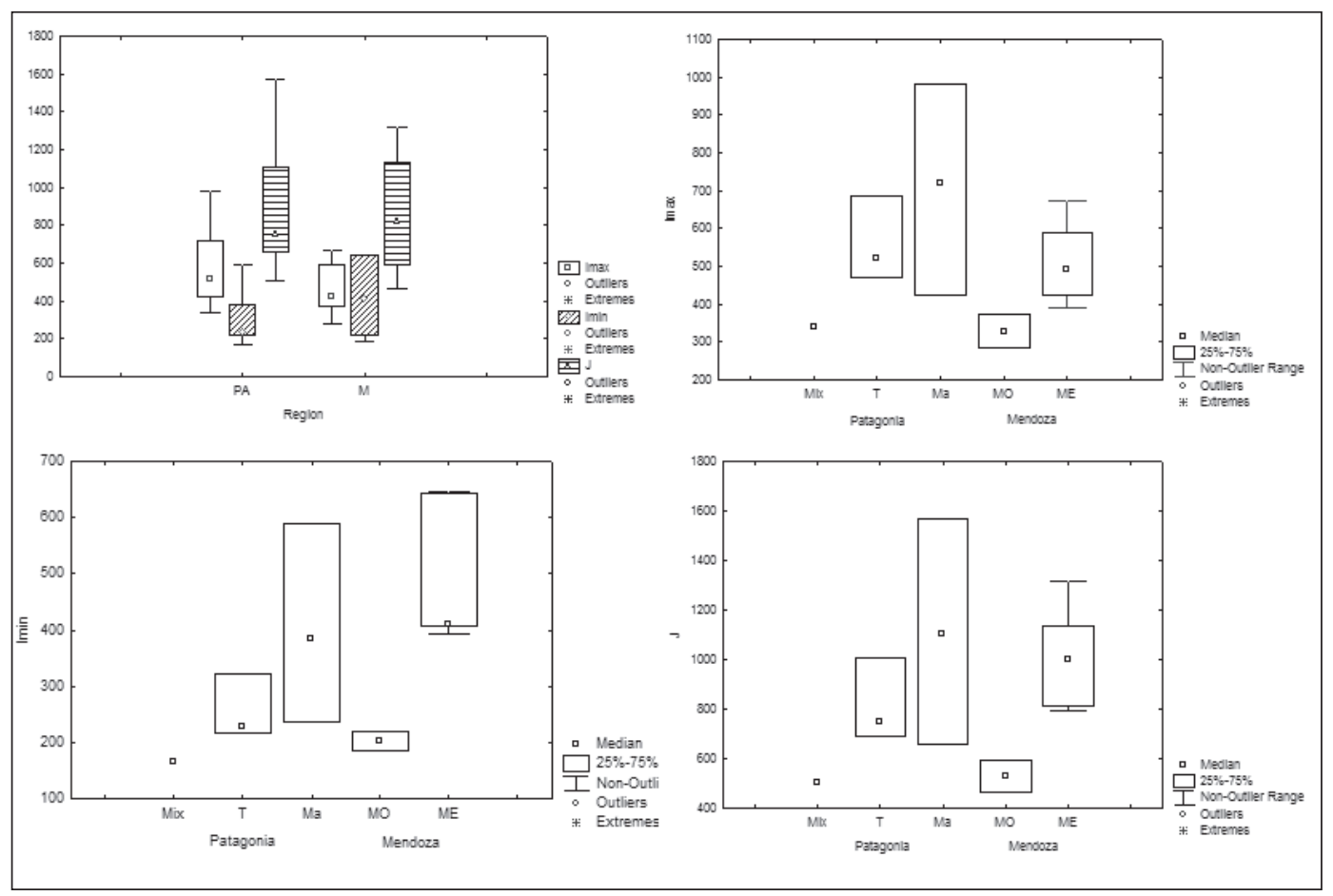

Fig. 3. Valores biomecánicos totales y comparativos de Imin, Imax y J (en $\mathrm{mm}^{4}$ ) entre individuos con dietas diferentes en Patagonia y Mendoza, obtenidos en 35\% de las tibias.

Respecto de los valores logrados en la totalidad de los conjuntos, no se observaron diferencias significativas en los indicadores biomecánicos obtenidos entre las muestras de Patagonia Austral y Mendoza. En ambos casos los valores de CA mostraron una correlación alta y positiva con los de TA, lo que indica que el espesor cortical aumenta de acuerdo al área total transversal en cada individuo, permitiendo asumir condiciones fisiológicas y nutricionales suficientes para sostener los requerimientos mecánicos. Sólo los restos del sitio arqueológico Paiashauaia, correspondiente a un individuo masculino adulto de la región del Canal Beagle, presentó una reducción considerable del área cortical en relación a la TA, que podría estar asociada a un trastorno metabólico sistémico (Suby et al., 2011). La similitud en los valores totales entre regiones podría indicar que la influencia de las diferencias ambientales y de actividad física podría no ser suficiente en estos casos para influir de manera significativa en la geometría seccional de las tibias.

Por el contrario, cuando los análisis fueron realizados clasificando las muestras de acuerdo a la actividad económica de los individuos, asumida a partir de análisis isotópicos, algunas tendencias, aunque no significativas en la mayoría de los casos, fueron identificadas en los patrones biomecánicos. En el caso de los restos de Patagonia Austral, se observó un mayor desarrollo de las tibias, en especial en las porciones distales, en aquellos individuos con dietas predominantemente marítimas, en relación a los individuos de dietas mixtas y terrestres. Estos resultados son concordantes con los presentados previamente por Stock (2006), Suby (2007) y Suby y Guichón (2009) a partir de análisis de la robusticidad de tibias y fémures, lo que indicaría que los individuos con este tipo de economías podrían haber desarrollado actividades que implicaran un mayor desarrollo morfológico óseo de los miembros inferiores. Sin embargo, los resultados contradicen los presentados por Pearson y Millones (2005), quienes sugieren que no habría evidencias de diferencias asociadas a la actividad física entre Yámanas y Selk'nam. El contraste entre los resultados podría estar 


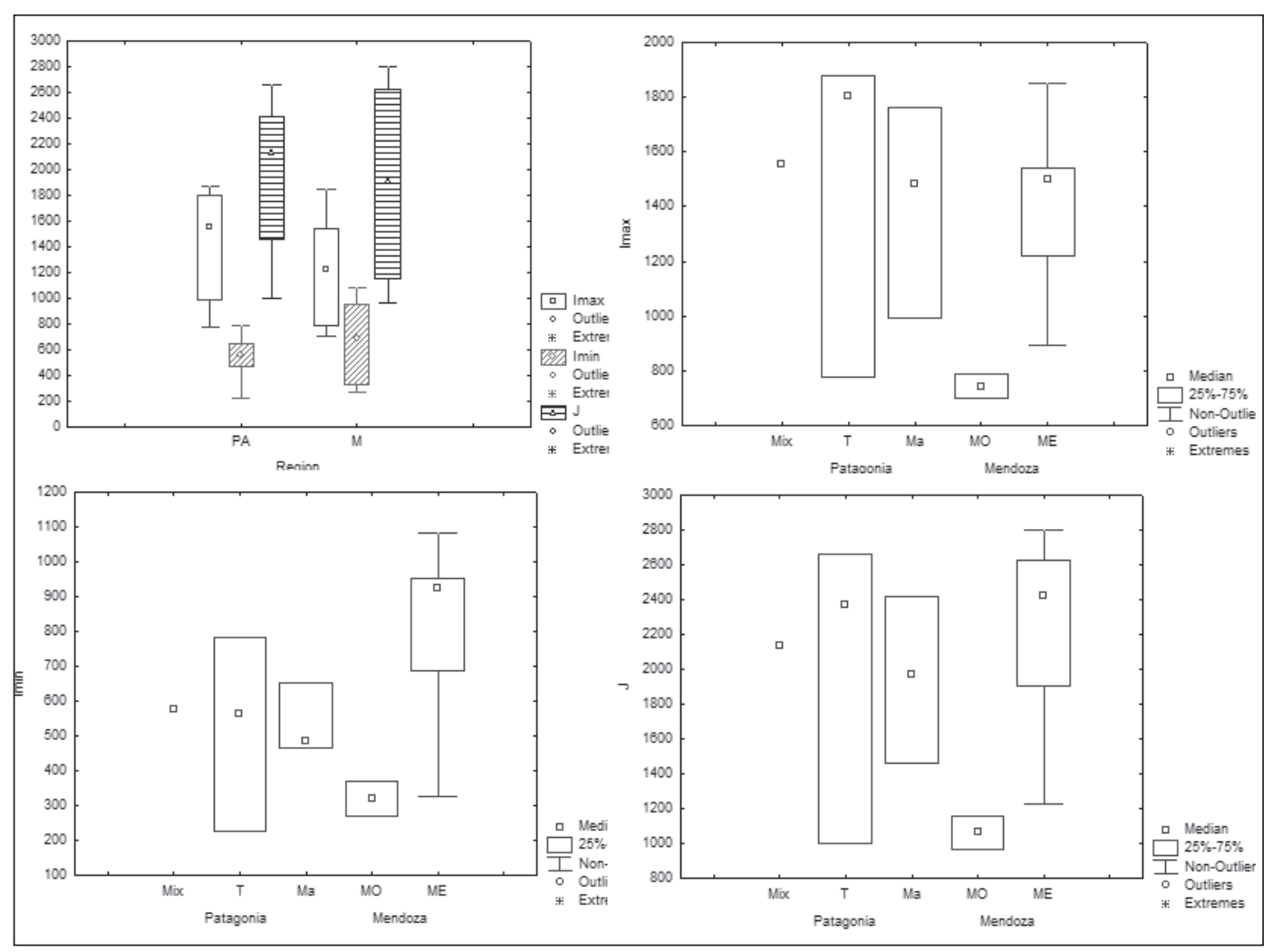

Fig. 4. Valores biomecánicos totales y comparativos entre individuos con dietas diferentes en Patagonia y Mendoza, obtenidos en $65 \%$ de las tibias.

asociado al bajo tamaño muestral analizado en ambos trabajos, o bien al sistema clasificatorio empleado sobre los conjuntos óseos, dado que las diferencias en la robusticidad podrían estar asociadas a los patrones económicos, a diferencias genéticas o a ambas variables. En este trabajo, consideramos que los patrones económicos podrían estar mejor expresados a partir de los resultados paleodietarios y no a partir de supuestos etnográficos asignados según las características contextuales y geográficas.

En el caso de los estudios sobre los individuos de la región Centro-Norte de Mendoza, aunque no presentaron una variación significativa con los restos de Patagonia Austral, si lo hicieron entre sí. Los individuos provenientes de la región montañosa de Mendoza, cuyos resultados isotópicos muestran un mayor consumo de maíz (Gil et al., 2009) presentaron valores biomecánicos que indican una menor robusticidad de las estructuras de las tibias, lo que podría reflejar una menor demanda física. Resultados biomecánicos similares fueron presentados en otras poblaciones cuyas economías se basaron en el consumo de maíz, especialmente en América del Norte (e.g. Bridges et al., 2000; Larsen, 2002), donde una menor movilidad y un empobrecimiento en la calidad y variedad de la dieta fue asociada a un menor desarrollo de las estructuras morfológicas de los miembros inferiores. Aunque un patrón similar se observó en los dos individuos de esta región analizados, cuyos valores de CA y TA son los menores de la totalidad de la muestra de Mendoza analizada en este trabajo, la relación entre ambas variables es similar a la que presentan los demás individuos (Fig. 2), por lo que es posible sugerir que las condiciones metabólicas en ambos casos permitieron sostener los requerimientos fisiológicos y mecánicos, lo que resulta concordante con las bajas prevalencias de indicadores de estrés identificadas en individuos de esta región (Novellino et al., 2004; Gil et al., 2009).

Los análisis realizados no estimaron la posi- 
ble influencia del dimorfismo sexual en los valores de los marcadores de robusticidad debido al reducido tamaño muestral Sin embargo, estudios previos consideraron que el sexo tiene un menor impacto en la morfología de los miembros inferiores que en los superiores entre sexos (Bridges 2000; Pearson y Lieberman 2004). En este sentido, no es posible descartar que parte de la variabilidad observada se deba al dimorfismo sexual o al posible impacto del género en el desarrollo de las estructuras óseas, asociados a distribución sexual de labores físicas que impliquen diferencias en las demandas mecánicas. Este aspecto deberá ser considerado en futuros análisis, evaluando no sólo elementos de los miembros inferiores sino también superiores, donde las variaciones debidas al sexo/género han sido mejor documentadas (Ruff y Larsen 2014).

Al igual que otros marcadores de estrés músculo-esqueletal, durante los últimos años se ha hecho especial énfasis en las dificultades de otorgar una asociación entre el mayor desarrollo de algunas estructuras óseas y el nivel de actividades generales y específicas de los individuos. Críticas fundamentadas, como la realizada recientemente por Jurmain et al. (2012) sugiere que los estudios del desarrollo de marcadores de estrés, entre ellos la geometría transversal de estructuras óseas, deben ser interpretados cautelosamente, considerando las múltiples causas atribuidas a su variabilidad a partir de datos clínicos. Factores genéticos, dietarios y metabólicos pueden contribuir al desarrollo óseo. En base a los estudios morfológicos realizados en restos del sur de Patagonia (Cocilovo y Guichón, 1986; Varela et al., 1993-94; Guichón, 1994), no es posible descartar posibles diferencias morfológicas esqueletales entre grupos debidas a variabilidad genética. Sin embargo, los resultados expuestos en este trabajo sugieren que los patrones dietarios y las posibles actividades económicas asociadas a ellos podrían explicar, al menos en parte, la mayor robusticidad de las estructuras óseas de las porciones distales de los miembros inferiores en algunos individuos. La relevancia de otros factores asociados deberán ser objeto de nuevos análisis que permitan profundizar las causas de la variabilidad intra e interpoblacional en el desarrollo óseo asociado a los patrones dietarios en el sur de Sudamérica.

\section{AGRADECIMIENTOS}

Agradecemos a los Dres. L. A. Borrero, F. A. Zangrando, E. L. Piana, M. Salemme y F. Santiago por brindarnos la posibilidad de estudiar los restos de Patagonia Austral considerados en este trabajo. Nuestro agradecimiento a los Dres. S. Costantino y C. Capiel del Instituto Radiológico Mar del Plata, por su constante colaboración en el análisis radiográfico de restos esqueletales. También al Dr. V. Durán por la lectura del manuscrito y sus comentarios. A dos revisores anónimos por sus comentarios, que ayudaron a mejorar el texto final.

\section{LITERATURA CITADA}

Barberena R. 2002. Los límites del mar. Buenos Aires: Sociedad Argentina de Antropología.

Borrero LA, Barberena R. 2006. Hunter-gatherer home ranges and marine resources. An archaeological case from Southern Patagonia. Curr Anthropol 47:855-867. doi: $10.1086 / 507186$

Brickley M, Ives R. 2008 The Bioarchaeology of Metabolic Bone Disease. San Diego: Academic Press.

Bridges PS, Blitz JH, Solano MC. 2000. Changes in long bone diaphyseal strength with horticultural intensification in west-central Illinois. Am J Phys Anthropol 112:217-238. doi:10.1002/(SICI)1096-8644

Brock SL, Ruff CB. 1988. Diachronic patterns of change in structural properties of the femur in the prehistoric American Southwest. Am J Phys Anthropol 75:113127. doi:10.1002/ajpa.1330750113

Buikstra JE, Milner GR. 1991. Isotopic and archaeological interpretations of diet in the central Mississippi valley. J Archaeol Sci 18:319-329. doi:10.1016/03054403(91)90068-Z

Cocilovo JA, Guichón RA. 1986. Propuesta para el estudio de las poblaciones aborígenes del extremo austral de Patagonia. An Inst Patagon 6:111-123.

Gil AF. 2003. Zea mays on South American periphery: chronology and dietary importance. Curr Anthropol 44:295-300.

Gil AF, Tykot R, Neme GA, Shelnut N. 2006. Maize on the frontier. Isotopic and macrobotanical data from CentralWestern Argentina. En: Staller J, Tykot R, Benz B, editores. Histories of maize. Multidisciplinary approaches to the prehistory, biogeography, domestication and evolution of maize. New York, Academic Press. p 199-214. doi:10.1086/367972

Gil AF, Neme GA, Tykot H, Novellino P, Cortegoso V, Duran V. 2009. Stable isotopes and maize consumption in Central Western Argentina. Inter J Osteoarchaeol 19:215-236. doi:10.1002/oa.1041

González Díaz E, Fauqué L. 1993. Geomorfología. En: Ramos V, editor. XII Congreso Geológico Argentino. Geología y Recursos Naturales de Mendoza. Relatorio I (14): Mendoza. p 217-234.

González-José R, García Moro C, Dahinten S, Hernández M. 2002. Origin of Fueguian-Patagonians: an approach to population history and structure using $\mathrm{R}$ matrix and matrix permutation methods. Am J Phys Anthropol 14:308-320. doi:10.1002/ajhb.1003 
Guichón RA. 1994. Antropología física de Tierra del Fuego. Caracterización biológica de las poblaciones prehispánicas. Tesis Doctoral Inédita. Facultad de Filosofía y Letras. Universidad de Buenos Aires.

Guichón RA, Suby JA. 2011. Estudio bioarqueológico de los restos humanos recuperados por Anne Chapman en Caleta Falsa, Tierra del Fuego. Magallania 39(1):163177. doi: $10.4067 / \mathrm{S} 0718-22442011000100010$

Guichón RA, Muñoz AS, Borrero LA. 2000. Datos para una tafonomía de restos óseos humanos en Bahía San Sebastián, Tierra del Fuego. Relaciones XXV:297-313.

Gusinde M. 1982. Los indios de Tierra del Fuego. Los Selk'nam. Buenos Aires: Centro Argentino de Etnología Americana.

Gusinde M. 1986. Los indios de Tierra del Fuego. Los Yamana. Buenos Aires: Centro Argentino de Etnología Americana.

Holliday TW. 1999. Brachial and crural indices of European late upper Paleolithic and Mesolithic humans. J. Hum. Evol. 36:549-566. doi:10.1006/jhev.1998.0289

Holt BM. 2003. Mobility in upper Paleolithic and Mesolithic Europe: evidence from the lower limb. Am J Phys Anthropol 122:200-215. doi:10.1002/ajpa.10256

Hyades P, Deniker J. 1891. Anthropologie et ethno-graphie. Mission scientifique du Cap Horn, 1882-1883. Vol. VII. Paris: Gauthier-Villars et fils.

Jurmain R, Cardoso FA, Henderson C, Villote S. 2012. Bioarchaeology's holy grail: the reconstruction of activity. En: Grauer A, editor. A companion to paleopathology. Malden: Willey-Balckwell. p 531-552.

Kelly RL. 1992. Mobility/sedentism: concepts, archaeological measures, and effects. Ann Rev Anthropol 21:43-66. doi:10.1146/annurev.an.21.100192.000355

Lalueza C, Perez-Perez A, Prats E, Cornudella L, Turbon D. 1997. Lack of founding amerindian mitochondrial DNA lineages in extinct aborigines from Tierra de Fuego-Patagonia. Hum Mol Gen 6:41-46. doi:10.1093/ $\mathrm{hmg} / 6.1 .41$

Larsen CS. 1995. Biological changes in human populations with agriculture. Ann Rev Anthrop 24:185-213. doi:10.1146/annurev.an.24.100195.001153

Larsen CS. 2002. Bioarchaeology: the lives and lifestyles of past people. J Archaeol Res 10(2):119-166. doi:10.1023/A:1015267705803

Ledger M, Holtzhausen LM, Constant D, Morris AG. 2000. Biomechanical beam analysis of long bones from a late 18th century slave cemetery in Cape Town, South Africa. Am J Phys Anthrop 112:207-216. doi:10.1002/(SICI)1096-8644(2000)112:2<207::AIDAJPA7>3.0.CO;2-K

L'Heureux GL, Barberena R. 2008. Evidencias bioarqueológicas en Patagonia meridional: el sitio Orejas de Burro 1 (Pali Aike, Provincia de Santa Cruz). Intersecc Antropol 9:11-24.

L'Heureux GL, Guichón RA, Barberena R, Borrero LA. 2003. Durmiendo bajo el faro. Estudio de un entierro humano en Cabo Vírgenes (C.V.17), Pcia. de Santa Cruz, República Argentina. Intersecc Antropol 4:87-98.

Macintosh AA, Davies TG, Ryan TM, Shaw CN, Stock JT. 2013. Periosteal versus true cross-sectional geometry: A comparison along humeral, femoral, and tibial diaphysis. Am J Phys Anthropol 150(3):442-452. doi:10.1002/ ajpa. 22218

Menéndez L, Novellino P, D’Addona L, Beguelín M, Brachetta N, Bernal V. 2014. En: Cortegoso V, Durán V, Gasco A, editores. Arqueología de ambientes de altura de Mendoza y San Juan, Argentina. Mendoza: EDIUC. p 101-125.
Morello F, Borrero LA, Massone M, Stern C, García-Herbst A, Mcculloch R, Arroyo-Kalin M, Calás E, Torres J, Prieto A. 2012. Hunter-gatherers, biogeographic barriers and the development of human settlement in Tierra del Fuego. Antiquity 86:71-87. doi:10.1017/ S0003598X00062463

Nikita E, Siew YY, Stock J, Mattingly D, Lahr MM. 2011. Activity patterns in the Sahara Desert: An interpretation based on cross-sectional geometric properties. Am J Phys Anthropol 146:423-434. doi:10.1002/ajpa.21597

Novellino P, Gil A, Neme G, Durán V. 2004. El consumo de maíz en el holoceno tardío del oeste argentino: isótopos estables y caries. Rev Esp Antropol Am 34:85-110. doi:10.5209/rev_REAA.2004.v34.24567

O’Neill MC, Ruff CB. 2004. Estimating human long bone cross-sectional geometric properties: a comparison of noninvasive methods. J Hum Evol 47(4):221-235. doi:10.1016/j.jhevol.2004.07.002

Orquera LA, Piana EL. 1999. La vida material y social de los Yámana. Buenos Aires: Eudeba.

Orquera LA, Piana EL. 2009. Sea nomads of the Beagle channel in southernmost South America: over six thousand years of coastal adaptation and stability. J Isl CoastArchaeol 4:61-81. doi:10.1080/15564890902789882

Panarello H, Zangrado AF, Tessone A, Kozameh L, Testa N. 2006. Análisis comparativo de paleodietas humanas entre la región del canal de Beagle y Península Mitre: perspectivas desde los isótopos estables. Magallania 34(2):37-47.

Pearson OM, Lieberman DE. 2004. The aging of Wolff's "law": ontogeny and responses to mechanical loading in cortical bone. Yrbk Phys Anthropol 47:63-99. doi:10.1002/ajpa.20155

Pearson O, Millones M. 2005. Rasgos esqueletales de adaptación al clima y a la actividad entre los habitantes aborígenes de Tierra del Fuego. Magallania 33(1):37-50. doi:10.4067/S0718-22442005000100003

Pearson OM, Buikstra JE. 2006. Behavior and the bones. En: Buikstra JE, Beck LA, editoras. Bioarchaeology. The contextual analysis of human remains. AmsterdamBoston: Elsevier.

Perez SI, Bernal V, González PN. 2007. Morphological differentiation of aboriginal human populations from Tierra del Fuego (Patagonia): implications for South American peopling. Am J Phys Anthropol 133:1067-1079. doi:10.1002/ajpa.20633

Rhodes JA, Knüsel CJ. 2005. Activity-related skeletal change in medieval humeri: cross-sectional and architectural alterations. Am J Phys Anthropol 128:536-546. doi:10.1002/ajpa.20147

Ruff CB. 2008. Biomechanical analyses of archaeological human skeletons. En: Katzenberg MA, Saunders SR, editores. Biological anthropology of the human skeleton. New York: Wiley Liss. p 183-206.

Ruff CB, Hayes WC. 1982. Subperiosteal expansion and cortical remodeling of the human femur and tibia with aging. Science 217:945-948.

Ruff CB, Hayes WC. 1983. Cross-sectional geometry of Pecos Pueblo femora and tibiae-a biomechanical investigation. I. Method and general patterns of variation. Am J Phys Anthropol 60:359-381. doi:10.1002/ ajpa.1330600308

Ruff CB, Larsen CS, Hayes WC. 1984. Structural changes in the femur with the transition to agriculture on the Georgia coast. Am J Phys Anthropol 64:125-136. doi:10.1002/ajpa.1330640205

Ruff CB, Larsen CS. 2014. Long bone structural analyses and the reconstruction of past mobility: a historical re- 
view. En: Carlson KJ, Marchi D, editors. Reconstructing mobility. New York: Springer. p 13-30.

Ruff CB, Trinkaus E, Walker A, Larsen CS. 1993. Postcranial robusticity in Homo, I: temporal trends and mechanical interpretation. Am J Phys Anthropol 91:21-53. doi:10.1002/ajpa.1330910103

Santiago F, Salemme M, Suby JA, Guichón RA. 2011. Restos óseos humanos en el norte de Tierra del Fuego. Aspectos contextuales, dietarios y paleopatológicos. Intersecc Antropol 12:147-162.

Sládek V, Berner M, Sailer R. 2006. Mobility in central European late eneolithic and early Bronze age: tibial cross-sectional geometry. J Archaeol Sci 33:470-482. doi:10.1002/ajpa.20372

Stock JT. 2006. Hunter-gatherer postcranial robusticity relative to patterns of mobility, climatic adaptation, and selection for tissue economy. Am J Phys Anthropol 131:194-204. doi:10.1002/ajpa.20398

Stock J, Pfeiffer S. 2001. Linking structural variability in long bone diaphyses to habitual behaviors: foragers from the southern African later Stone age and the Andaman islands. Am J Phys Anthropol 115:337-348. doi:10.1002/ajpa.1090

Stock JT, Shaw CN. 2007. Which measures of diaphyseal robusticity are robust? A comparison of external methods of quantifying the strength of long bone diaphyses to cross-sectional geometric properties. Am J Phys Anthropol 134:412-423. doi:10.1002/ajpa.20686

Suby JA. 2007. Propiedades estructurales de restos óseos humanos y paleopatología en Patagonia Austral. Tesis Doctoral Inédita. Facultad de Ciencias Exactas y Naturales. Universidad Nacional de Mar del Plata.

Suby JA, Guichón RA. 2009. Diet, nutrition and paleopathology in Southern Patagonia. Some experiences and perspectives. Int J Osteoarchaeol 19:328-336. doi:10.1002/oa.1040
Suby JA, Salemme M, Santiago F. 2008. Análisis paleopatológico de los restos humanos del sitio Puesto Pescador 1 (Tierra del Fuego). Magallania 36(1):53-64.

Suby JA, Zangrando AF, Piana EL. 2011. Exploraciones osteológicas de la salud de las poblaciones humanas del Canal Beagle. Relaciones XXXVI:249-270.

Trinkaus E. 1981. Neanderthal limb proportions and cold adaptation. En: Stringer CB, editor. Aspects of human evolution. Londres: Taylor \& Francis. p. $187-$ 224.

Trinkaus E, Ruff C. 1999a. Diaphyseal cross-sectional geometry of near eastern middle Palaeolithic humans: the femur. J Archaeol Sci 26:409-24. doi:10.1006/ jasc. 1998.0343

Trinkaus E, Ruff C. 1999b. Diaphyseal cross-sectional geometry of near eastern middle Paleolithic humans: The tibia. J Archaeol Sci 26:1289-1300. doi:10.1006/ jasc.1998.0361

Varela H, Cocilovo A, Guichón RA. 1993-94. Evaluaciones de la información somatométrica por Gusinde sobre los aborígenes de Tierra del Fuego. Anales Instituto Patagonia 22:193-205.

Weiss E. 2005. Humeral cross-sectional morphology from 18th century Quebec prisoners of war: limits to activity reconstruction. Am J Phys Anthropol 126:311-317. doi:10.1002/ajpa.20069

White T, Black M, Folkens P. 2012. Human osteology. San Diego: Academic Press.

Yesner DR, Figuerero Torres MJ, Guichón RA, Borrero LA. 2003. Stable isotope analysis of human bone and ethnohistoric subsistence patterns in Tierra del Fuego. J Anthropol Archaeol 22: 279-291. doi:10.1016/S02784165(03)00040-0

Zangrando AF. 2009. Historia evolutiva y subsistencia de cazadores-recolectores marítimos de Tierra del Fuego. Buenos Aires: Sociedad Argentina de Antropología. 